\title{
Trilepton Signal of Grand Unified Models at the Tevatron
}

\author{
E. Accomando, R. Arnowitt and B. Dutta \\ Center for Theoretical Physics, Department of Physics, Texas AEM University, College Station, TX 7r843-4242
}

(November, 1998)

\begin{abstract}
At the Tevatron, the most promising channel to detect supersymmetry is three leptons plus missing energy, where the leptons are $e$ 's and/or $\mu$ 's. This final state appears from the production of chargino and second lighetst neutralino. However in grand unified models with universal scalar masses at the grand unified scale, this final state mostly consists of $\tau$ 's which are hard to detect. We show that for some regions of non universality in the scalar masses at the GUT scale based on unifying groups like $\mathrm{SU}(5)$ or $\mathrm{SO}(10)$, the final state mostly consist of $3 l+E_{T}$ and $\tau l l+E_{T}$. The first mode has very high detection efficiency and the second one is expected to have high detection efficency as well. We also show that these models can have enough events in these modes to be detected in RUN II.
\end{abstract}

] Due to its many attractive features, supersymmetry (SUSY) has become the main focus of experimental search. Among the existing colliders, the upcoming RUN II $\left(\sqrt{s}=2 \mathrm{TeV}\right.$ and $2 f b^{-1}$ luminosity $)$ of the Tevatron is going to have the highest reach in terms of scanning the supersymmetric parameter space. Consequently, final states of different SUSY particles are being examined on the basis of their detectibility.

The production of the lightest chargino and the second lightest neutralino $\left(\chi_{1}^{ \pm}, \chi_{2}^{0}\right)$ is found to be very promising for the discovery of SUSY. In most of the supersymmetric models, for large regions of the parameter space, the masses of $\chi_{1}^{ \pm}$and $\chi_{2}^{0}$ are within the reach of RUN II. The final states of this mode can involve three leptons $+E_{T}$. If these trileptons are $e$ 's and $\mu$ 's, then the detection of the final states becomes easier since $e, \mu$ have large detection efficiencies $(\sim 85 \%)$. If, however, the final states involve $\tau$ 's, the detection becomes harder, since the $\tau$ detection efficiency has not yet been specified. The $\tau$ can be detected hadronically ('thin jet') or leptonically. If we depend on the leptonic modes (since we are working in a machine with lots of jets), then the effective leptonic cross section of the final state with multi $\tau$ 's becomes very small (due to the small leptonic branching ratio of the $\tau$ ).

The trilepton channel at the Tevatron has been analysed by many groups in various different supersymmetric models [1.2. These theoretical scenarios range from the supergravity motivated models (SUGRA) to Gauge Mediated SUSY breaking Models (GMSB). In absence of experimental evidence as well as a full understanding of the dynamics of SUSY breaking, no unique model has as yet emerged. Models where the different SUSY masses are related, are found to be more compelling due to their predictivity. Among these, the SUGRA models with the constraint that all the scalar masses are same (and gaugino masses also the same) at the grand unified theory scale $M_{G}$ (universal boundary conditions) is the most popular one. The major finding of the trilepton search analysis in this model is that the $\tau$ dominated final states are more abundant for large as well as for smaller $\tan \beta$ [3]. GMSB models give rise to hard photons (easy to detect) or high $p_{T} \tau$ in the final states of the $\left(\chi_{1}^{ \pm}, \chi_{2}^{0}\right)$ production.

In this letter, we will examine the chargino-neutralino $\left(\chi_{1}^{ \pm}, \chi_{2}^{0}\right)$ pair production in grand unified models within the framework of SUGRA with radiative breaking of the electroweak symmetry. We will not impose universality of the scalar masses at $M_{G}$. Instead, we will impose the constraints on the masses based on the representations of the groups to which these fields belong and will use unifying groups like $\mathrm{SU}(5)$ and $\mathrm{SO}(10)$. We find that these non universal boundary conditions may lead to specific and detectable characteristics in the trilepton final state. In parts of the parameter space the final state infact is dominated by $3 l+E_{T}(l$ is $e, \mu)$ or $\tau l l+E_{T}$ instead of all $\tau$. Due to the high detection efficiency $3 l$ mode is the best candidate and due to high luminosity, one can have a lower threshold to trigger them via a dilepton trigger $l l+E_{T}$. In the same fashion the $\tau l l$ mode is also expected to have high detection efficiency [4]. Analysing the trilepton signal in various channels in RUN II, this general boundary condition (non-universal scenario) may be distinguished from the universal one in wide regions of the parameter space.

In supergravity motivated unified models, the non-universality at the boundary can appear naturally. A general non-flat Kahler metric (where the SUSY breaking field is coupled to the observable fields with different couplings) can induce non-universalities in the scalar masses [5]. Since the Higgs sector is weakly constrained by the requirement of FCNC suppression and the third generation is only weakly coupled to the FCNC processes, one may assume that the third generation squark, slepton and Higgs masses are non-universal at the GUT scale, while the first and second generation scalar masses and the gaugino masses are assumed to be universal. (Non universalities in the gaugino sector can be also induced, but are small in most models, and so we assume these masses to be universal). Scalar 
mass non universalities also can be generated from the running of the RGE's from the Planck scale or string scale to the GUT scale. In this case, because of the quark-lepton unification, not only the third generation squark masses, but the third generation slepton masses will be different from the other generation masses [6]. Finally, we mention that non universalities can be generated from the so-called D-terms arising from the rank reduction of the groups which embed the SM as a subgroup, when the GUT group has ranks higher than the SM .

Let us examine the parametrization of the non-universalities. The Higgs soft breaking masses are given by $m_{H_{1}}^{2}=$ $m_{0}^{2}\left(1+\delta_{1}\right) ; m_{H_{2}}^{2}=m_{0}^{2}\left(1+\delta_{2}\right)$. The third generation fermion soft breaking masses are as follows: $m_{q_{L}}^{2}=m_{0}^{2}\left(1+\delta_{3}\right)$; $m_{u_{R}}^{2}=m_{0}^{2}\left(1+\delta_{4}\right) m_{e_{R}}^{2}=m_{0}^{2}\left(1+\delta_{5}\right) ; m_{d_{R}}^{2}=m_{0}^{2}\left(1+\delta_{6}\right) ; m_{l_{L}}^{2}=m_{0}^{2}\left(1+\delta_{7}\right)$. The $\delta_{i}$ exhibit the amount of non universality. If we use a unifying group, where the fields belong to some represantaion of that group the $\delta_{i}$ develop relations among themselves. For example, in the case of the GUT group SU(5) the matter fields are embedded in $\overline{5}$ and 10 representations. The $\delta_{i}$ in the previous expressions have the following relations: $\delta_{3}=\delta_{4}=\delta_{5}=\delta_{10} ; \quad \delta_{6}=\delta_{7}=\delta_{\overline{5}}$.

Any GUT group which has an SU(5) with the matter fields in the 10 and $\overline{5}$ representations will have the above pattern of non universalities. In the case of $S O(10)$, if we demand a direct breaking into the SM and keep the $5+\overline{5}$ Higgs in the same 10 of $S O(10)$, we get an additional constraint, $\delta_{5}=\delta_{2}-\delta_{1}$. In this note we will use $\delta_{1}, \delta_{2}, \delta_{\overline{5}}$ and $\delta_{10}$ to represent the nonuniversalities. One need not be restricted in this choice.

In order to determine the physical masses we need to know $\mu$ which is determined from the electroweak symmetry breaking condition. For $\tan \beta$ small enough, one obtains the following analytical expression [7]: $\mu^{2}=\mu_{\text {univ }}^{2}+\frac{m_{0}^{2}}{t^{2}-1}\left(\delta_{1}-\right.$ $\left.\delta_{2} t^{2}-\frac{D_{0}-1}{2}\left(\delta_{2}+2 \delta_{10}\right) t^{2}\right)+\frac{3}{5} \frac{t^{2}+1}{t^{2}-1} S_{0} p+$ oneloop, where $t=\tan \beta, D_{0} \simeq 1-\left(m_{t} / 200 \sin \beta\right)^{2}, S_{0}=\operatorname{Tr}\left(Y m^{2}\right), \mathrm{p}=0.0446$ and $\mu_{\text {univ }}^{2}$ is the remaining universal part.

Let us first discuss the trilepton signals in the case of universal boundary conditions. It has been shown that [3] among all the trilepton plus $E_{T}$ modes, only the $3 \tau+E_{T}$ production cross section is large in most of the parameter space when the sleptons are produced on shell. Among the other modes, only $\tau l l$ becomes comparable but only for the very small region of $\tan \beta \leq 5$. The branching ratio (BR) in this mode decreases rapidly and at $\tan \beta=10$, for e.g. $m_{0}=100 \mathrm{GeV}$ and $m_{1 / 2}=200 \mathrm{GeV}$, the $\tau l l$ mode becomes about $\frac{1}{10}$ of the BR of $3 \tau$ mode (The BR of $3 \tau$ remains almost the same). The $\tau l l$ cross section becomes $21.2 \mathrm{fb}$. The situation worsens by decreasing $m_{0}$. For example, at $m_{0}=30 \mathrm{GeV}$ (keeping the other parameters the same) the cross section for the $\tau l l$ mode becomes $11 \mathrm{fb}$ (and in this region the BR of $\tau \tau l$ becomes large). Even when the sleptons become offshell, the branching ratio to the leptonic modes involving multi $\tau$ 's is large. The $\tau$ domination in the signal (onshell or offshell case) is usually expected when $\tan \beta$ is large. But the domination seems to persist even in the region of low $\tan \beta$.

The reason for this misfortune depends primarily on two factors. The lighter stau $\left(\tilde{\tau}_{1}\right)$ mass is lighter than the selectron mass. In the onshell case, the decay width depends on $\left(\Delta m^{2}\right)^{2}$ (where $\Delta m^{2}$ is the mass ${ }^{2}$ difference between the gaugino and the slepton), which is larger for the modes involving $\tau$. The other factor is that the $\chi_{2}^{0}$ is primarily a wino which has coupling to the left sleptons only which are heavier than both the right handed selectrons and the $\chi_{2}^{0}$. On the otherhand, the lighter $\tilde{\tau}_{1}$ is a mixture of $\tilde{\tau}_{L}$ and $\tilde{\tau}_{R}$ due to the large left right mixing $m_{\tau} \mu \tan \beta$. (We will assume $A=0$ at the GUT scale throughout the analysis, but a non-zero value will not change the conclusion). Consequently, $\chi_{2}^{0}$ primarily will decay into the lighter $\tilde{\tau}_{1}$ and a $\tau$. Among these two factors, the first one has a larger impact. The $\chi_{1}^{ \pm}$is a mixture of charged Higgsino and wino giving rise to a dominating $\tilde{\tau}_{1} \nu$ final state when the $\tilde{\tau}_{1}$ is onshell.

In the case of non-universal boundary conditions, new effects can reduce the right-handed selectron mass and raise the $\tilde{\tau}_{1}$ mass (and can make it even larger than the selectron mass) and the BR into the $3 l$ mode is no longer suppressed. The magnitude of $\mu^{2}$ can also be decreased which increases the $\tilde{\tau}_{1}$ mass and thereby decreases the BR into $\tau$ dominated final states. Finally, the nature of $\chi_{2}^{0}$ can change with the change in the size of $\mu$ (the wino component can decrease and the bino component can increase).

Another important point to note is that, when we use the non universal boundary conditions we have to add the term $S \equiv \alpha_{1} \frac{3 Y_{i}}{10 \pi} \sum_{i}\left(Y_{i} m_{i}^{2}\right)$ to the RGEs of the fields. The contribution from this term is zero in the case of universal boundary condition but is non zero in the non-universal case.

We are now ready to discuss the results. In Fig.1 we show the masses as a function of the nonuniversalities. In Fig.1a we plot the $\chi_{1}^{ \pm}, \tilde{\tau}_{1}, \tilde{\nu}_{L}, \tilde{e}_{R}$ and $\mu$ as functions of $\delta_{10}$. The other $\delta_{i}$ are zero. We see that the $\tilde{\tau}_{1}$ mass increases as $\delta_{10}$ increases. Since $\delta_{10}$ does not contribute to the $\mathrm{S}$ term, the $\tilde{e}_{R}$ or $\tilde{\nu}_{L}$ masses do not get any effects. The $\chi_{2}^{0}$ mass is very close to the $\chi^{ \pm}$mass.

In Fig. $1 \mathrm{~b}$ we plot the the same masses as functions of $\delta_{2}$. Since $\delta_{2}$ contributes to the $\mathrm{S}$ term, we see that the $\tilde{e}_{R}$ mass decreases for the positive values of $\delta_{2}$. The $\tilde{\tau}_{1}$ mass also decreases up to moderate values of $\tan \beta$, but the decrease is slightly lower than the $\tilde{e}_{R}$ mass which helps to raise the raise the $3 l$ or $\tau l l$ BRs. This happens because, as $\delta_{2}$ increases, $\mu$ gets reduced which reduces the off diagonal element in the stau mass matrix. The reduction in the size of $\mu$ also lowers the $\chi_{1}^{ \pm}$and $\chi_{2}^{0}$ masses. In the large $\tan \beta$ case $\left(\tan \beta \gtrsim 25\right.$, depending on the size of $\left.\delta_{2}\right)$ the $\tilde{\tau}_{1}$ mass is increased which helps to increase the $3 l$ or $\tau l l$ BRs. 
In the Figs.2 and 3, we plot the production cross sections of the leptonic modes as function of $m_{0}$. We have chosen a pattern of non-universality which obeys the $S U(5)$ group structure. We have used $\delta_{1}=-0.5, \delta_{2}=0.5, \delta_{\overline{5}}=1$ and $\delta_{10}=0.5$. These values of the $\delta_{i}$ also allow the simplest $\mathrm{SO}(10)$ breaking patterns $(\mathrm{SO}(10) \rightarrow \mathrm{SM})$ at $M_{G}$ i.e. the condition $\delta_{\overline{5}}=\delta_{2}-\delta_{1}$ is satisfied.

In Fig.2a, we use $\tan \beta=10, m_{1 / 2}=200 \mathrm{GeV}$ and $\mu>0$. The sign convention we adopt is the same as in ref [8]. In Fig.2b we plot the same parameter space for the universal boundary condition. We observe the following:

a) For $30 \lesssim m_{0} \lesssim 70 \mathrm{GeV}$, the $3 \tau$ and $\tau \tau l$ mode dominate initially in the non-universal case. But the $\tau l l$ mode is not far behind and as $m_{0}$ increases the BR in this mode increases. The reason for $\tau$ domination in this region (the $\tilde{e}_{R}$ and $\tilde{\tau}_{1}$ masses are almost same) is due to the Wino nature of $\chi_{2}^{0}$. In this region the chargino can decay into $l \tilde{\nu}_{l}$. As the sneutrino mass goes offshell towards the end of the region, the branching ratios of the $3 l$ and the $\tau \tau l$ modes reduce. In the universal case (Fig $2 \mathrm{~b}$ ), the $3 \tau$ mode dominates with the $\tau l l$ and the $\tau \tau l$ modes coming next.

b) For $70 \lesssim m_{0} \lesssim 100 \mathrm{GeV}$, the $\tau l l$ and $3 \tau$ mode dominate in Fig.2a, and the $3 l$ mode starts becoming significant. In this region the selectron mass becomes lower than the $\tilde{\tau}_{1}$ mass and hence the BR of $\chi_{2}^{0}$ into $e$ 's and $\mu$ 's increases. Towards the end of the region, the sleptons (first $\tilde{\tau}_{1}$ ) becomes offshell. In the universal case the $3 \tau$ mode is dominant in this region.

c) For $100 \lesssim m_{0} \lesssim 200 \mathrm{GeV}$, the sleptons are mostly offshell. In Fig. 2 a the $3 l$ is the dominant decay mode and next to that is the $\tau l l$ mode. The production cross section decreases as we increase $m_{0}$. At the end of this region the $3 \tau$ and $\tau \tau l$ modes increase again due to the offshell Higgs contribution. In the universal case the $3 \tau$ mode dominates initially. For $m_{0} \geq 130$, the $3 l$ mode becomes equal to the $3 \tau$ mode, but the cross section is very reduced by that time. (The $\tau \tau l$ mode dominates here).

Using a dilepton trigger, and if we use 5 events in RUN II as a bench mark for a SUSY signal (corresponding to the cross section of $25 \mathrm{fb}$ with $10 \%$ acceptance rate [9]), the inclusive $l l+E_{T}$ production allow us to scan $50 \lesssim m_{0} \lesssim 130$ $\mathrm{GeV}$. In the universal case we do not get 5 events in this mode for any value of $m_{0}$. In the case of a $\tau$ l trigger, (assuming the same acceptance rate) the inclusive $\tau l+E_{T}$ production allows us to scan $30 \lesssim m_{0} \lesssim 100 \mathrm{GeV}$. Here again, in the universal case we do not find any $m_{0}$ value which gives rise to 5 events.

If we reduce $m_{1 / 2}$, the BRs of the $\tau l l$ and the $3 l$ mode increase more because of the increase in phase space. In Fig.3 we plot the cross sections of the leptonic modes as function of $m_{0}$ for $m_{1 / 2}=150 \mathrm{GeV}$ and $\mu>0$.

In the case of $\mu<0$, the $\chi_{1}^{ \pm} \chi_{2}^{0}$ production cross section decreases due to increased chargino and neutralino masses. We find that this choice of the $\mu$ sign gives rise to a scenario analogous to what one could get by increasing the value of $m_{1 / 2}$. The contribution of the on shell sneutrino for $m_{0} \lesssim 80 \mathrm{GeV}\left(m_{1 / 2}=200 \mathrm{GeV}\right)$ enhances the branching of the chargino into leptons. The $3 \tau$ channel shrinks.

We have discussed above the dependence of the leptonic signal on $m_{1 / 2}$ and $m_{0}$ at a fixed value of $\tan \beta$ and for a particular set of $\delta_{i}$. We next examine the correlation between $\tan \beta$ and the non-universal boundary conditions and find that, even for moderate to large values of $\tan \beta$, the abundance of $\tau$ 's in the final state can be reduced in favor of the $3 l$ and $\tau l l$ channels for positive $\delta_{10}$ and $\delta_{5}$.

Fig. 4 shows a parametric plot of the $3 l$ and $\tau l l$ with the cross section fixed at $35 \mathrm{fb}$ as functions of $\delta_{10}$ and $\tan \beta$. Here we have used $\delta_{2}=0.5, \delta_{1}=-0.5, \delta_{5}=1, m_{0}=100 \mathrm{GeV}, m_{1 / 2}=200 \mathrm{GeV}$ and $\mu>0$. Both $\delta_{5}$ and $\delta_{10}$ help to raise $\tilde{\tau}_{1}$ mass. Since $\delta_{10}$ affects the right stau mass, it has a larger impact in increasing $\tilde{\tau}_{1}$ mass and thereby decreasing the branching ratio of the $\tau \tau l$ and $3 \tau$ modes. The selectron mass is reduced by a small amount (a few GeV) through the $\mathrm{S}$ term. Hence an increment of $\delta_{10}$ will raise the $\mathrm{BR}$ of the $3 l$ and $\tau l l$ mode. It is evident from the figure that as $\tan \beta$ increases, larger values of $\delta_{10}$ are needed in order to compensate the decreasing of the $\tilde{\tau}_{1}$ mass. However, one sees that for $\delta_{10} \leq 2$, the $3 l$ mode is large even for $\tan \beta \simeq 25$.

An increase in the magnitude of $\delta_{2}$ raises the $3 l$ and $2 l$ BRs. (The bino component of $\chi_{2}^{0}$ increases.) But since the production cross section becomes small (the coupling becomes smaller because the wino component of the $\chi_{2}^{0}$ reduces), the net increase in the cross sections of the same modes is small. Hence a change in $\delta_{2}$ does not affect the cross sections of the leptonic modes much.

One can use other decay chains of $\mathrm{SO}(10)$, e.g. $S O(10) \rightarrow S U(3)_{c} \times S U(2)_{L} \times S U(2)_{R} \times U(1)_{B-L}$. In this case again the $\tilde{\tau}_{1}$ mass can be increased by increasing the $m_{\tilde{\tau} R}^{2}$ mass by adding a non universality. This term can affect the $\tilde{e}_{R}$ through the $\mathrm{S}$ term, but the effect is small. One can also cancel this effect by adding an identical magnitude of non universality to $m_{\tilde{\tau} L}^{2}$. The net effct is the $\tilde{\tau}_{1}$ will increase further without changing the $\tilde{e}_{R}$ mass.

To conclude, we have looked into the final states of the chargino-second lightest neutralino production at the Tevatron for $\sqrt{s}=2 \mathrm{TeV}$. In the models with universal boundary conditions the $3 \tau+E_{T}$ mode dominates among all the leptonic final states even for low values of $\tan \beta$. We have found that in grand unified models with non universal boundary conditions, the $3 l$ and $\tau l l$ final states can dominate over the $3 \tau$ or the $\tau \tau l$ modes for low and intermediate values of $\tan \beta$ for some regions of non universality. The magnitudes of the non universalities can be relatively small in order to give rise to the above type of scanarios. This result holds for either sign of $\mu$. Since the $3 l$ mode has by 
far the best detection efficiency, these nonuniversal boundary conditions may be tested in RUN II.

Work of E. A. and R. A have been supported by the National Science Foundation grant No. 9722090 We thank Teruki Kamon for his critical comments and suggestions. We also thank J.P. Done for useful discussions.

[1] R. Arnowitt and P. Nath, Mod. Phys. Lett. A2 (1987) 331; H. Baer and X. Tata, Phys. Rev. D47 (1993) 2739 ; J. Lopez et al., Phys. Rev. D48 (1993) 2062; T. Kamon, J. Lopez, P. McIntyre and J.T. White, Phys. Rev. D50 (1994) 5676; S. Mrenna, G. Kane, G.D. Kribs and J.D. Wells, Phys. Rev. D53 (1996) 1168; H. Baer, C. Kao and X. Tata, Phys. Rev. D48 (1993) 5175;D52 (1995) 1565; H. Baer, C-H. Chen, F. Paige and X. Tata, Phys. Rev. D54 (1996) 5866; H. Baer, C-H. Chen, M. Drees and F. Paige, Phys. Rev. D58 (1998) 075008.

[2] S. Dimopoulos, S. Thomas and J. D. Wells, Nucl. Phys. B488, (1997) 39; H. Baer, M. Brhlik, C-H. Chen and X. Tata, Phys. Rev. D55 (1997) 4463; S. Ambrosanio, G.L. Kane, G. D. Kribs, S. P. Martin and S. Mrenna, Phys. Rev. D54 (1996) 5395; J. L. Feng and Takeo Moroi, Phys. Rev. D58, (1998) 035001; B. Dutta and S. Nandi, hep-ph/9709511; B. Dutta, D.J. Muller and S. Nandi, hep-ph/9807390.

[3] V. Barger, C. Kao and T-J. Li, Phys. Lett. B424, (1998) 69.

[4] Private communication with Teruki Kamon.

[5] S.K. Soni and H.A. Weldon, Phys. Lett. B126 (1983) 215; V.S. Kaplunovsky and J. Luis, Phys. Lett. B306 (1993) 268.

[6] R. Barbieri, L.J. Hall, Phys. Lett. B338 (1994) 212; N. Polonski and A. Pomarol, Phys. Rev. D51 (1995) 6532.

[7] R. Arnowitt and P. Nath, Phys. Rev. D56 (1997) 2820;D46 (1992) 3981; J. Ellis and F. Zwirner, Nucl. Phys. B338 (1990) 317.

[8] H. E. Haber and G.L. Kane, Phys. Rept. 117,75,1985.

[9] F. Abe et al., Phys. Rev. Lett. 80, (1998)5280. 

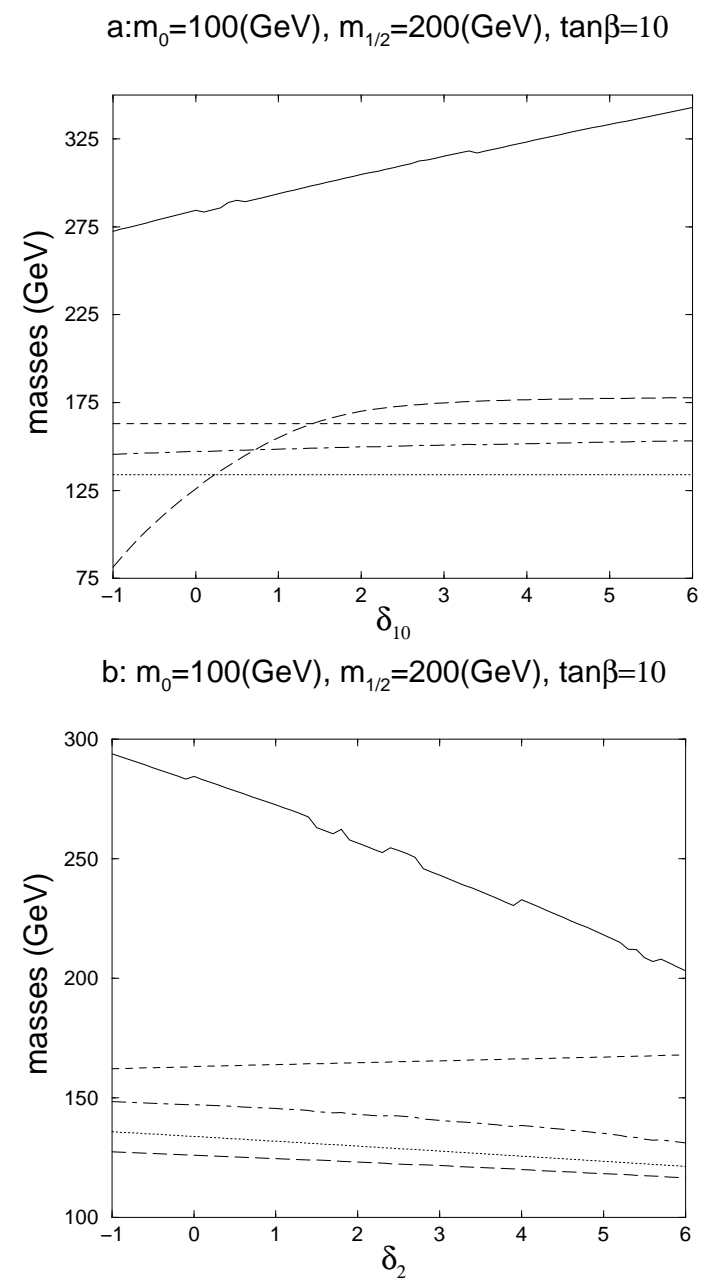

FIG. 1. $\mu$ (solid line), $\tilde{\tau}_{1}$ (long dashed), $\tilde{\nu}_{L}$ (short dashed), $\tilde{e}_{R}$ (dotted) and $\chi_{1}^{ \pm}$(dot dashed) are shown 


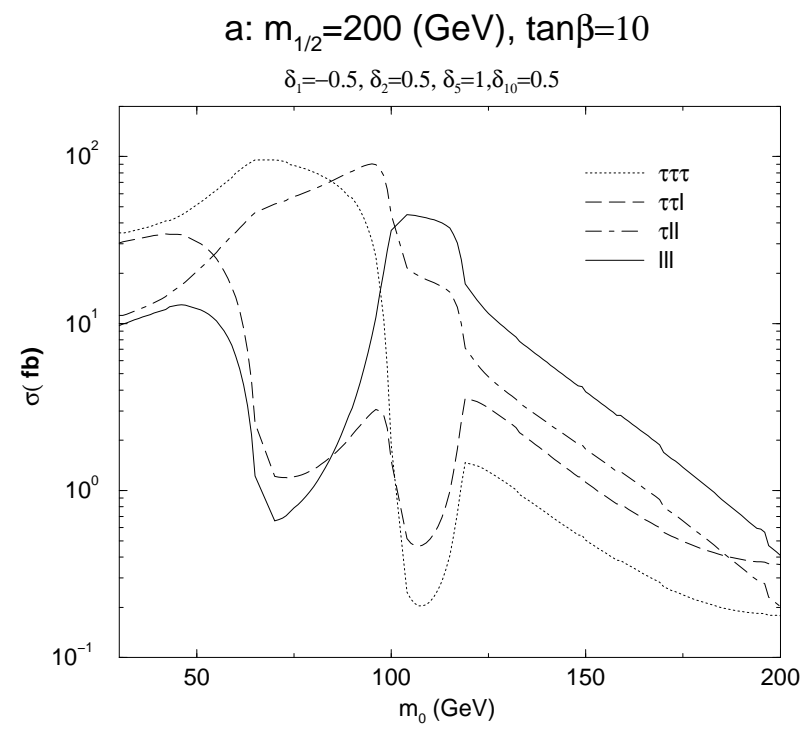

b: $m_{1 / 2}=200(\mathrm{GeV}), \tan \beta=10$

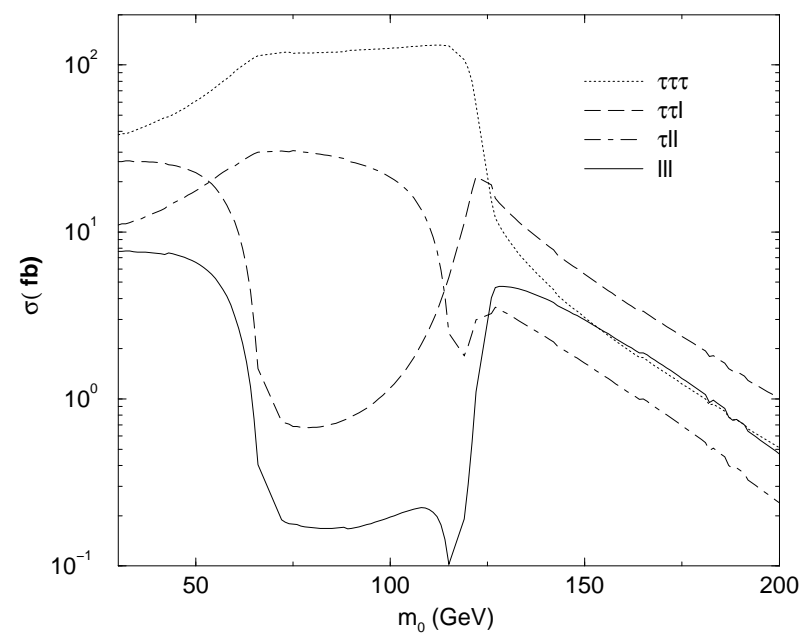

FIG. 2. Cross sections for all the leptonic modes. a)Non-universal case and b) universal case. 


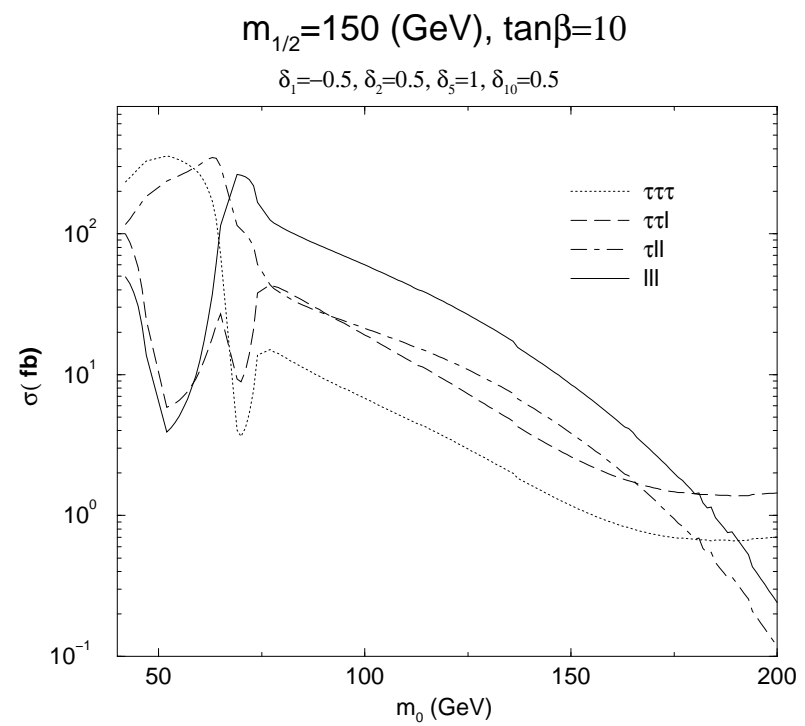

FIG. 3. Cross sections for all leptonic modesfor the non-universal case

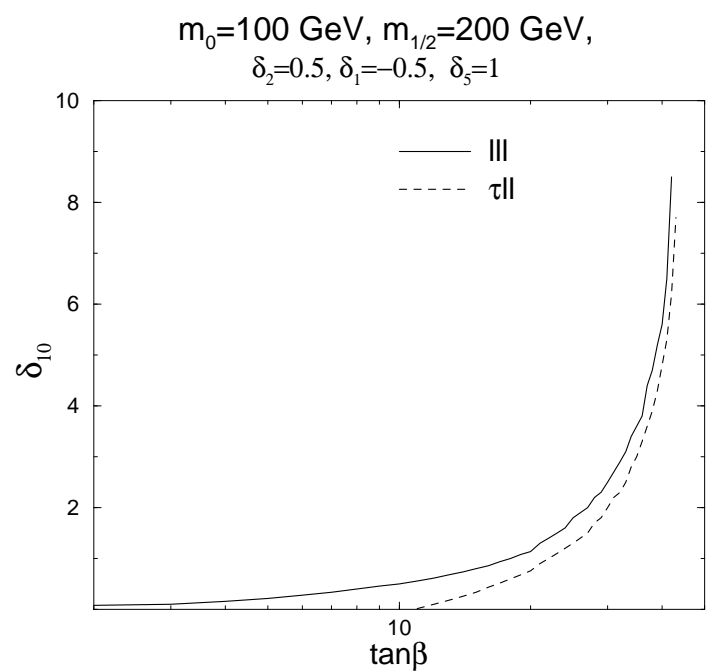

FIG. 4. The value of $\delta_{10}$ required as a fuction of $\tan \beta$ so that the production cross section for the $3 l$ and the $\tau l l$ modes be $35 \mathrm{fb}$. 